\title{
Viewing-as Explanations and Ontic Dependence
}

\author{
William D'Alessandro
}

\begin{abstract}
According to a widespread view in metaphysics and philosophy of science (the "Dependence Thesis"), all explanations involve relations of ontic dependence between the items appearing in the explanandum and the items appearing in the explanans. I argue that a family of mathematical cases, which I call "viewing-as explanations", are incompatible with the Dependence Thesis. These cases, I claim, feature genuine explanations that aren't supported by ontic dependence relations. Hence the thesis isn't true in general. The first part of the paper defends this claim and discusses its significance. I argue, for example, that many mathematical explanations are apparently compatible with Dependence, so the existence of counterexamples is interesting and non-obvious. The second part of the paper considers whether viewing-as explanations occur in the empirical sciences, focusing on the case of so-called fictional models (such as Bohr's model of the atom). It's sometimes suggested that fictional models can be explanatory even though they fail to represent actual worldly dependence relations. Whether or not such models explain, I suggest, depends on whether we think scientific explanations necessarily give information relevant to intervention and control.
\end{abstract}

This paper is about a certain mathematical phenomenon, and its implications for a widely held view about the metaphysics of explanation. I'll say more about the mathematical phenomenon shortly. The widely held view is this:

Dependence Thesis: All explanations reflect relations of ontic dependence between the items appearing in the explanandum and the items appearing in the explanans.

I plan to argue that a family of mathematical cases, which I'll call "viewing-as explanations", are incompatible with the Dependence Thesis. These cases, I claim, feature genuine explanations that aren't supported by ontic dependence relations. Hence the Dependence Thesis - which has achieved something approaching consensus status in metaphysics, philosophy of science and elsewhere -is not true in general.

The paper is organized as follows. $§ 1$ briefly describes the tension between Dependence and viewing-as explanations, and $\S 2$ presents some further examples from the mathematical literature. $§ 3$ looks more closely at the Dependence Thesis, the challenge posed by the mathematical cases, and several possible responses to the challenge. $\S 4$ considers whether viewing-as explanations are possible in the empirical sciences, focusing on the case of so-called fictional models. $§ 5$ briefly argues that the counterfactual approach to explanation also has trouble accommodating view-as cases. Finally, I offer some concluding remarks in $\S 6$.

\section{Setting up the problem}

It may seem strange to turn to pure mathematics for insights about explanation, since the literature is mostly populated with examples from metaphysics and empirical science. This is beginning to change; for instance, [Lange 2016] and [Reutlinger \& Saatsi forthcoming] are recent high-profile works that engage extensively with noncausal explanations, in mathematics and elsewhere. But there's more progress to be made on this front, and "intramathematical" explanation in particular deserves more attention than it gets. ${ }^{1}$ I hope this paper will help show that mathematics and the mainstream explanation debate have plenty to say to one another.

\footnotetext{
${ }^{1}$ Recent works on the subject include [Steiner 1978], [Resnik \& Kushner 1987], [Detlefsen 1988], [Sandborg 1998], [Hafner \& Mancosu 2005], [Harari 2008], [Mancosu 2008], [Lange 2014], [Pincock 2015], [D'Alessandro 2017], [Lange 2017].
} 
Here, then, is an example of my target phenomenon. Suppose you want to show that the sum of the first $n$ odd natural numbers is $n^{2}$. One approach is to use mathematical induction: clearly the statement is true for $n=1$, and applying the induction hypothesis gives

$$
\begin{aligned}
\sum_{k=1}^{n+1}(2 k-1) & =\sum_{k=1}^{n}(2 k-1)+2(n+1)-1 \\
& =n^{2}+2 n+1 \\
& =(n+1)^{2},
\end{aligned}
$$

as needed.

This gets the job done, logically speaking; the proof is certainly sound. But it seems not to explain the result. The fact that we can shuffle $1 \mathrm{~s}$ and $2 \mathrm{~s}$ and $n$ s around until they assume the right form gives no clue about why sums of odd numbers might have anything to do with perfect squares. (Indeed, in the view of many mathematicians and philosophers, inductive arguments like this one are generally unexplanatory; cf. [Lange 2009].)

Here's a different sort of proof, the key idea of which is to view numbers as arrangements of dots. Start with 1 , the first odd number, which can be regarded as a square array of side 1 (and hence of area $1^{2}$ ). The second odd number is 3 , and we can think of adding 3 to 1 as augmenting the original square array so as to make a new one of side 2 (and hence of area $2^{2}$ ). Similarly, adding 5 to $1+3$ gives a square array of side 3 , and so on, as in the diagram.

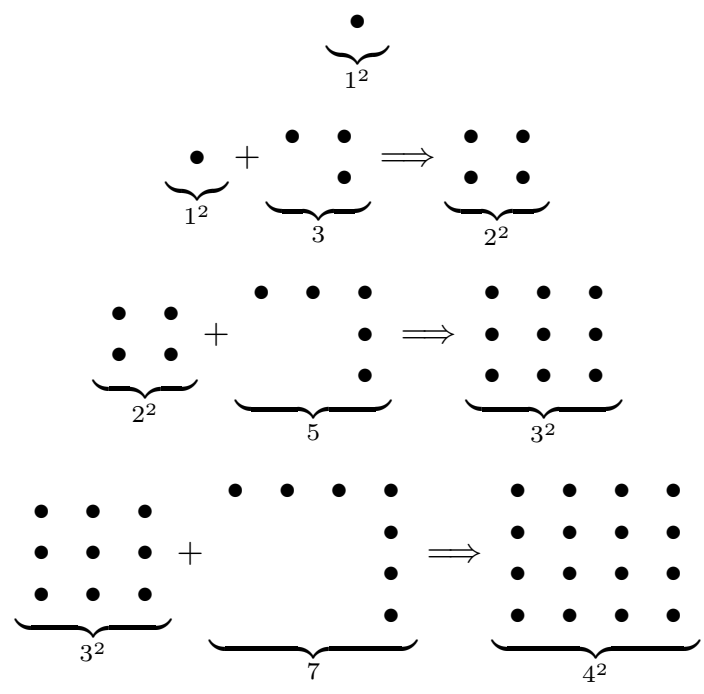

It's easy to see that the pattern will hold in general - for any $n-1$, the sum of the first $n-1$ odd numbers corresponds to a square array of side $n-1$ and area $(n-1)^{2}$, and adding the next odd number can be viewed as augmenting this array to produce a new one of side $n$ and area $n^{2}$. Hence the diagram shows that the sum of the first $n$ odd natural numbers is $n^{2}$. What's more, this sort of proof is plausibly explanatory. ${ }^{2}$ It shows that a fact about natural numbers - namely, the fact that $\sum_{k=1}^{n}(2 k-1)=n^{2}$-is explained by a fact about arrangements of dots - namely, the fact that certain augmentations of square arrays are themselves square arrays of the appropriate size. Call this case Numbers-Dots.

Numbers-Dots illustrates a common practice. Often, and for a variety of reasons, mathematicians view one mathematical object as another. Viewing-as frequently has explanatory benefits, as in the above case: by viewing numbers as arrangements of dots, we're put in a position to explain why the sum of the first $n$ odd numbers is $n^{2}$. And this identity seemed hard to account for otherwise.

\footnotetext{
${ }^{2}$ [Gullberg 1997] gives a similar dot-diagram proof that the sum of the first $n$ natural numbers is $n(n+1) / 2$, and claims that "the figure shows why" the identity holds (289). [Hanna 1990] contrasts the inductive proof of this identity with the dot-diagram proof, claiming that the latter but not the former is explanatory (10-11). [Steiner 1978] makes the same comparison with the same conclusion (136-137). See chapter 8 of [Giaquinto 2007] for a discussion of the epistemology of dot-diagram arguments, including a thorough defense of the claim that the images used in such arguments count as genuine proofs.
} 
It's not hard to see why some viewing-as explanations might seem to conflict with the Dependence Thesis. After all, Dependence says that explanatory relationships are always grounded in ontic dependence relations between the relevant entities. But viewing-as explanations need not satisfy this condition. Numbers, after all, evidently aren't arrays of dots, nor are they composed of them, constituted by them or causally influenced by them. The two classes of object are similar in certain ways, but mere similarity is neither a form of dependence nor an explanation-backing relation. So it's hard to see how to reconcile Dependence with cases like Numbers-Dots. (Of course, it remains to be shown that this tension is actually insoluble. There may be some other, less obvious form of dependence at work in such cases. Or perhaps there's some way of analyzing the cases that removes the appearance of conflict. These possibilities will be discussed at length in $\S 3$ below.)

Philosophers have yet to look carefully at viewing-as and its role in explanation, but the phenomenon hasn't gone entirely unnoticed. Indeed, it appears already in Mark Steiner's landmark 1978 paper, which kicked off the recent literature on explanation in mathematics. Steiner mentions "Hardy's explanation of the lawless behavior of a certain numerical function [obtained] by regarding it, a la Ramanujan, as a 'snapshot' at each $n$ of the resultant of infinitely many sine waves of incompatible periods and decreasing amplitudes" ([Steiner 1978], 148). (Unfortunately Steiner doesn't discuss this case, or the general phenomenon, in detail.)

Questions about the metaphysical underpinnings of mathematical explanation aren't limited to cases of viewing-as, of course. What it takes for one piece of mathematics to explain another remains a difficult open question. But it's worth noting that many kinds of mathematical explanation seem compatible with Dependence. Consider the following, for instance.

1. It's often claimed that some mathematical properties are relatively natural or fundamental while others are relatively unnatural or derivative. ${ }^{3}$ Assuming this is true, there's plausibly an asymmetrical dependence of some less natural properties on more natural ones. For instance, the graph of a quadratic polynomial $f(x)$ crosses the $x$-axis twice just in case $f(x)$ has two distinct real zeroes. But the latter property is arguably more fundamental than the former; it seems correct to say that the graph of $f(x)$ crosses the $x$-axis twice because $f(x)$ has two real zeroes, but wrong to attribute the existence of the zeroes to the behavior of the graph. ${ }^{4}$

2. Many philosophers hold that sets depend ontologically on their members. ${ }^{5}$ As Øystein Linnebo writes: "According to the prevailing iterative conception, sets are 'formed from' their elements. The relation between a set and its elements is thus asymmetric, because the elements must be 'available' before the set can be formed, whereas the set need not be, and indeed cannot be, 'available' before its elements are formed. A set thus appears to depend on its elements in a way in which the elements do not depend on the set" ([Linnebo 2008], 72). The same presumably goes for at least some other set-theoretic structures.

3. It might also be, as some proponents of structuralism think ${ }^{6}$, that the reverse pattern of dependence sometimes holds - i.e., that some mathematical objects depend on the structures to which they belong. Consider abstract groups, for instance, whose elements have no determinate properties except those imposed on them by the group multiplication table. Since the elements can't be characterized or identified independently of the group, it's plausible that the former depend ontologically on the latter. (Cf. [Linnebo 2008], section VI.)

4. Some mathematical explanations that don't involve set membership may nevertheless feature other "composition"- or "building"-style relations. ${ }^{7}$ For instance, we can often explain properties of natural numbers in terms of properties of their prime factors, and this is plausibly because numbers are in

\footnotetext{
${ }^{3}$ See for instance [Maddy 2000], [Corfield 2005], [Tappenden 2008], [Lange 2015].

${ }^{4} \mathrm{~A}$ more sophisticated example: in a discussion of the Hadwiger conjecture in graph theory, Paul Seymour says that "the real reason" why a given graph is 4-colorable may be that it contains no $K_{5}$ minor ([Seymour 2016], 418). This suggests that the latter property is more fundamental, and that it provides the metaphysical ground for 4-colorability.

${ }^{5}$ E.g. [Fine 1995], and many of the subsequent contributors to the literature on metaphysical grounding.

${ }^{6}$ E.g. [Resnik 1981] and [Shapiro 1997].

${ }^{7}$ The notion of building is Karen Bennett's; cf. [Bennett 2017]. For more on the relationship between set membership and other kinds of composition, see [Fine 2010].
} 
some sense "composed of primes". (As the mathematician Jordan Ellenberg writes, "primes are the atoms of number theory, the basic indivisible entities of which all numbers are made" ([Ellenberg 2014], 139).) Similar building-like relations might be thought to hold between a finite group and its simple composition factors, a polynomial and its irreducible factors, and likewise for any object that admits factorization (especially when the factors are unique).

5. Chris Pincock has proposed "a new kind of dependence relation that can obtain in the absence of any essential composition relation" ([Pincock 2015], 11), which he calls "abstract dependence". Abstract dependence is closely related to instancehood. Roughly, an object $X$ abstractly depends on an object $Y$ just in case $X$ is an instance of $Y$, and $Y$ is in fact the least abstract object having $X$ as an instance (in the sense that there's no intermediate object $Z$ such that $X$ is an instance of $Z$ and $Z$ is an instance of $Y$ ). Pincock argues that some mathematical explanations are backed by abstract dependence relations. For instance, he thinks polynomial equations metaphysically depend on their Galois groups, which is why facts about groups can explain facts about the solvability of equations. ("What makes a given polynomial equation solvable, we should say, is that its Galois group is solvable" ([Pincock 2015], 11).)

Each of these cases, it seems, involves ontological dependence of a type that's suitable for underwriting explanations. ${ }^{8}$ So it's far from true that mathematical explanations in general are incompatible with the Dependence Thesis. If there are mathematical counterexamples to the thesis, as I plan to argue, then the existence of such cases is interesting and non-obvious.

The next section presents several cases of this sort. Before moving on, however, let me fix some terminology. When an object $X$ is being viewed as an object $Y$, I'll refer to $X$ as the source object and to $Y$ as the target object. Also, following standard mathematical practice, I'll use the phrases "view $X$ as $Y$ ", "regard $X$ as $Y$ ", "think of $X$ as $Y$ ", and "interpret $X$ as $Y$ " interchangeably. It's worth noting, however,that "seeing $X$ as $Y$ " traditionally has a different, distinctively perceptual meaning. The classic case of seeing-as, or "aspect perception", is Wittgenstein's duck-rabbit ${ }^{9}$, and the literature on this phenomenon has centered on questions of attention, perceptual content and the like. ${ }^{10}$ Although seeing-as and viewing-as are similar in some respects, and although seeing-as has its place in mathematical cognition $^{11}$, I take the two phenomena to be importantly distinct. (For instance, viewing-as sometimes involves perception or visualization, but it needn't do so in general.) I intend to focus on viewing-as here.

There's a great deal to be said about the nature of viewing-as and its role in mathematical practice. Unfortunately I can't say much about these broader issues here. For readers wondering how the viewing-as phenomenon fits into mainstream cognitive theory, however, it's perhaps worth mentioning that the notion of frames offers a promising starting point. ${ }^{12}$ In particular, viewing an object $X$ as an object $Y$ is something very much like adopting $Y$ as a frame for thinking about $X$. This sort of cognition is useful in mathematics, as in science, because frames often structure thought in beneficial ways - by imposing useful taxonomies, raising important facts to salience, introducing comparisons whose consequences can be pursued in fruitful directions, and so on. For more on the varieties of frames deployed in scientific inquiry and the cognitive benefits they confer, see Elisabeth Camp's enlightening recent work ([Camp forthcoming]).

\footnotetext{
${ }^{8}$ I don't mean to take a stand on the genuineness of each of these purported dependence relations, or on the significance of the associated forms of mathematical explanation. My point here is just that many cases of mathematical explanation are plausibly underwritten by one or another type of metaphysical dependence.

${ }^{9}$ See Part II, section xi of [Wittgenstein 2009].

${ }^{10}$ For recent work on seeing-as, see for instance [Day \& Krebs 2010], [Kemp \& Mras 2016] and [Beaney et al. forthcoming]. Although the usage I've mentioned is standard, seeing-as is sometimes taken to encompass non-perceptual "perspectival" phenomena like metaphor, as in [Camp 2008].

${ }^{11}$ See for instance [Coliva 2012] on the role of seeing-as in reasoning with Euclidean diagrams, and [Floyd 2010] on Wittgenstein's ideas about seeing-as in logic, probability and other mathematical subjects.

${ }^{12}$ The notion of frame has a long history in psychology and the social sciences, the general idea being that of an interpretative system that structures thought, perception or communication in some domain. Earlier research on frames focused mostly on their (often negative) effects in the social, political and economic spheres. The phenomena at issue include stereotypes (cf. [Herald 2010]), "spin" in politics and journalism (cf. [Entman 2007]), psychological effects involving people's (often seemingly irrational) sensitivity to differing descriptions of a decision problem (cf. [Tversky \& Kahneman 1981]), and the like.
} 


\section{Viewing-as explanations: some cases}

At the outset of the paper, I presented a case in which viewing one thing (numbers) as another (arrays of dots) led to an explanatory insight (about why the sum of the first $n$ odd numbers is $n^{2}$ ). That case is typical of the kind of explanation I want to discuss. But further examples will, I hope, help make the phenomenon more vivid. So I'll present some additional cases, again drawn from the mathematical literature, that exemplify the link between viewing-as and explanation.

Consider first an example discussed by Timothy Gowers, concerning a famous identity due to Euler:

$$
\sum_{n=1}^{\infty} \frac{1}{n^{2}}=1+\frac{1}{4}+\frac{1}{9}+\frac{1}{16}+\cdots=\frac{\pi^{2}}{6}
$$

As Gowers notes, this looks mysterious at first: "What on earth, one might wonder, has $\pi$ to do with adding up reciprocals of squares? This is a perfectly legitimate question" ([Gowers 2008], 261). He then shows how to answer the question using ideas from Fourier analysis, in the following way. First, given a periodic function $f: \mathbb{R} \rightarrow \mathbb{C}$ with period $2 \pi$, define the $n$th Fourier coefficient of $f$-denoted $a_{n}$-by the formula

$$
a_{n}:=\frac{1}{2 \pi} \int_{-\pi}^{\pi} f(x) e^{i n x} d x .
$$

Then the theorem known as Plancherel's identity describes the sum of the squared moduli of the Fourier coefficients:

$$
\frac{1}{2 \pi} \int_{-\pi}^{\pi}|f(x)|^{2} d x=\sum_{n=-\infty}^{\infty}\left|a_{n}\right|^{2}
$$

Now let $f$ be the function such that $f(x)=1$ when $\left(2 n-\frac{1}{2}\right) \pi \leq x \leq\left(2 n+\frac{1}{2}\right) \pi$ for some integer $n$, and $f(x)=0$ otherwise. (Note that $f$ has period $2 \pi$.) Combining Plancherel's identity with some calculation and manipulation, we get the formula

$$
\begin{aligned}
\frac{\pi^{2}}{8} & =1+\frac{1}{3^{2}}+\frac{1}{5^{2}}+\frac{1}{7^{2}}+\cdots \\
& =\sum_{n=1}^{\infty} \frac{1}{n^{2}}-\sum_{n=1}^{\infty} \frac{1}{(2 n)^{2}} \\
& =\frac{3}{4} \sum_{n=1}^{\infty} \frac{1}{n^{2}} .
\end{aligned}
$$

Finally, multiplying both sides by $\frac{4}{3}$ gives the original statement, $\sum_{n=1}^{\infty} \frac{1}{n^{2}}=\frac{\pi^{2}}{6}$. After going through this argument, Gowers writes:

Now we have a reason for the appearance of $\pi$ : it comes up in the formula for the Fourier coefficients. What is more, its appearance there can be explained as well. A periodic function on $\mathbb{R}$ is more naturally thought of as a function defined on the unit circle. The Fourier coefficient $a_{n}$ is a certain average defined on the unit circle, so we have to divide by the length of the circle, which is $2 \pi$. (262)

Gowers' reasoning, then, seems to be as follows. We wanted to know at first why $\pi$ shows up in the identity $\sum_{n=1}^{\infty} \frac{1}{n^{2}}=\frac{\pi^{2}}{6}$. This turns out to involve a result from Fourier analysis: namely, the fact that Plancherel's theorem applied to a certain periodic function $f$ gives a closely related identity involving $\pi$ and reciprocals of squares. What's more, we can explain why $\pi$ appears here by viewing $f$ as a function $g$ defined on the unit circle, and noting that the Fourier coefficients are a type of average involving the values of $g$. Since taking this average involves dividing by the circumference of the circle, the appearance of $\pi$ is no surprise.

This looks like a case where viewing-as makes a crucial contribution to a mathematical explanation. The function $f$ is defined to have domain $\mathbb{R}$ and range $\mathbb{C}$, and it's not obvious what $\pi$ has to do with averaging a function like that. It's only when we regard $f$ as a quite different object - the function $g$ defined on the unit circle - that we find the explanation we were after. 
A second example comes from Laptev and Rozenfel'd's account of developments in

nineteenth-century geometry. The relevant passage occurs during a discussion of Lobachevky's discovery of hyperbolic geometry, and in particular the efforts by him and others to prove its consistency. Lobachevsky himself was only partially successful at this. But later authors, notably Poincaré and Minkowski, discovered an interpretation of the hyperbolic plane that not only showed it to be consistent, but also explained certain similarities between hyperbolic and spherical geometry. As Laptev and Rozenfel'd write:

$[T]$ he geometry of the hyperbolic plane [with Gaussian curvature $-\frac{1}{q^{2}}$ ] can be realized on a sphere of imaginary radius $q i$ in a subspace of complex space, whose points have rectangular $x$ and $y$-coordinates that are real $(x=\bar{x}, y=\bar{y})$, and $z$-coordinates that are purely imaginary $(z=-\bar{z})$. This subspace can be regarded as a real affine space in which the distance $d$ between the points with rectangular coordinates $x_{1}, y_{1}, z_{1}$ and $x_{2}, y_{2}, z_{2}$ is defined by the formula

$$
d^{2}=\left(x_{2}-x_{1}\right)^{2}+\left(y_{2}-y_{1}\right)^{2}+\left(z_{2}-z_{1}\right)^{2} .
$$

...The sphere of radius $q i$ in this space has the form of a hyperboloid of two sheets (the geometry of the hyperbolic plane is realized on each nappe of such a hyperboloid...)... This interpretation, which demonstrates vividly the consistency of Lobachevskii's planimetry, explains why the formulas of hyperbolic trigonometry can be obtained from those of spherical trigonometry by replacing the radius of the sphere by qi. ([Laptev \& Rozenfel'd 1996], 64)

The formulas referred to here include the law of cosines and the law of sines, which in spherical geometry take the forms

$$
\begin{gathered}
\cos \frac{a}{r}=\cos \frac{b}{r} \cos \frac{c}{r}+\sin \frac{b}{r} \sin \frac{c}{r} \cos A, \\
\frac{\sin A}{\sin (a / r)}=\frac{\sin B}{\sin (b / r)}=\frac{\sin C}{\sin (c / r)}
\end{gathered}
$$

(where $a, b, c$ are the side lengths of a triangle, $A$ is the angle opposite $a$, and $r$ is the radius of the sphere). Lobachevky of course noticed the resemblance between the spherical and hyperbolic formulas, but he lacked the resources to completely understand it - doing so requires Poncelet's later idea of enriching Euclidean space with imaginary points. In the resulting "pseudo-Euclidean space", the notion of a sphere of radius qi makes sense, and one can view the hyperbolic plane as a hemisphere of such a "pseudosphere" (which is realized as a sheet or "nappe" of a hyperboloid). This relationship holds because a sphere of radius $r$ has Gaussian curvature $\frac{1}{r^{2}}$, whereas a hyperbolic plane has negative Gaussian curvature, so a sphere of imaginary radius corresponds to a hyperbolic surface. Thinking of the hyperbolic plane in this way, then, lets us explain the peculiar form taken by the laws of hyperbolic trigonometry.

Third and finally, here's a case in which visualization plays a key role, from Marc Konvisser's linear algebra textbook. The problem Konvisser starts with is that of finding the solutions to polynomial equations of the form $x^{n}-1=0$. For values of $n$ less than 5 , the solutions can be found by relatively simple algebraic methods (e.g., factoring or applying the quadratic formula). After displaying these solutions, Konvisser writes:

We have now found all the roots for all equations of the form $0=x^{n}-1$ for $n=1,2,3$, and 4 . However, this has given us little insight into how to find the solutions of such equations for $n \geq 5$. To see what is happening, we view complex numbers as vectors. ([Konvisser 1986], 25)

Konvisser then explains how addition and multiplication of complex numbers are to be understood on this interpretation, noting in particular that multiplying a complex number by $i$ corresponds to rotating the associated vector counterclockwise by an angle of $\pi / 2$. He continues:

Now let us see how this interpretation of multiplication by $i$ as rotation by $\pi / 2$ can help us solve our original problem of finding the roots of equations of the form $0=x^{n}-1 \ldots$ In order to find complex numbers that satisfy the equation $z^{n}=1$, let us see if we can find complex numbers $z$ so that multiplication by $z$ represents a rotation of $1 / n$ way around, that is, a rotation of $2 \pi / n .(29)$ 
Some simple trigonometry leads to the desired general result: "Let $z=\cos \theta+i \sin \theta$. Multiplication by $z$ represents a counterclockwise rotation by the angle $\theta$ " (31). This essentially solves the original problem. We wanted to find the solutions to $x^{n}-1=0$, i.e. the complex numbers $z$ corresponding to rotations of $2 \pi / n$. By the previous proposition, one such solution is $z=\cos (2 \pi / n)+i \sin (2 \pi / n)$. In fact, $z$ is a "primitive $n$th root of unity", and the remaining roots are the powers $z^{2}, z^{3}, \ldots, z^{n-1}$.

Clearly this is a case where viewing one kind of thing (complex numbers) as another (vectors in the plane) yields a satisfying solution to a problem about the original objects. Although Konvisser doesn't explicitly frame the situation in terms of explanation, he does point out the lack of insight afforded by the naïve algebraic approach, and he says that viewing complex numbers as vectors allows us to better "see what is happening" with respect to the roots of $x^{n}-1$. I think these remarks are plausibly understood as claims about the relative explanatoriness of the viewing-as strategy. In particular, I take it that facts about $1 / n$th rotation vectors explain why the solutions to $x^{n}-1=0$ have the form that they do.

It wouldn't be hard to add further examples to this list, and no doubt doing so would paint a richer picture. But hopefully the cases discussed so far are enough to give a good idea of the phenomenon. The next section looks more closely at the conflict between viewing-as explanations and the Dependence Thesis.

\section{$3 \quad$ Viewing-as, explanation and dependence}

The Dependence Thesis, recall, is the following claim:

Dependence Thesis: All explanations reflect relations of ontic dependence between the items appearing in the explanandum and the items appearing in the explanans.

This idea is at least partly descended from Wesley Salmon's program in philosophy of science, which insisted on the centrality of causal relations to explanation. ${ }^{13}$ But Dependence is now widely accepted in metaphysics and elsewhere (usually with the understanding that non-causal grounding relations are also fair game). Here's a sample, by no means exhaustive, of recent endorsements of Dependence-like claims: ${ }^{14}$

- "Explanations work only in virtue of the determinative relations that exist in the world. ...[W]e explain something by showing what makes it or what is responsible for it" ([Ruben 1990], 231).

- "My main proposal... is this: explanations track dependence relations. The relation that 'grounds' the relation between an explanans, $G$, and its explanatory conclusion, $E$, is that of dependence; namely, $G$ is an explanans of $E$ just in case $e$, the event being explained, depends on $g$, the event invoked" ([Kim 1994], 68).

- "[A]n explanation, when successful, captures or represents... an underlying real-world relation of dependence of some sort which obtains among the phenomena cited in the explanation in question" ([Koslicki 2012], 212, quoted in [Pincock 2015], 7).

- "[E]xplanations correctly identify features of the ontic structures that produce, underlie, or [are] otherwise responsible for the explanandum phenomenon" ([Craver 2014], 39).

- "Explanations provide information about relations of ontic dependence, causal and non-causal" ([Povich 2016], 14).

- "I offer a speculation: the relations that back explanation are the relations of directed dependency" ([Schaffer 2016], 83).

\footnotetext{
${ }^{13}$ See [Salmon 1984] for an early articulation of Salmon's "ontic conception". [Salmon 1989] describes how the ontic conception emerged from dissatisfaction with Hempel's deductive-nomological theory.

${ }^{14}$ It may be reasonable to read some of these authors as implicitly talking only about certain kinds of explanation (although I don't think that's the case across the board). Even though the conclusion of this paper may not directly contradict those authors' views, it should put pressure on them to clarify what we can and can't expect from their version of Dependence. (Thanks to an anonymous referee for prompting me to make this clearer.)
} 
As I've suggested, there's an apparent conflict between the Dependence Thesis and viewing-as explanations like those discussed above. That's because there generally aren't any obvious dependence relations between the source and target objects in such cases.

Of course we shouldn't expect mathematical explanations to involve causal dependence relations. But neither do the viewing-as cases feature familiar metaphysical relations like composition, constitution or realization. Sums of odd numbers aren't comprised of arrays of dots in any sense, nor is "array of dots" a functional role that numbers could realize. On the whole, there seems to be no sense in which dot arrays "make" or are "responsible for" numbers or their properties. Nevertheless, the viewing-as cases seem to involve genuine explanatory connections between the source and target objects. What conclusions should we draw from this?

There are, it seems, four main views one might take. The first possibility is that the viewing-as cases don't really involve genuine explanations, so they aren't counterexamples to the Dependence Thesis. The second and third possibilities hold that the cases do involve explanation, but they do so in ways that don't threaten Dependence. According to the second option, the explanantia in such cases are something other than the target objects. So the lack of determination relations between the sources and targets is no problem for Dependence. According to the third option, it's indeed true that facts about the target objects explain facts about the source objects - however, there are also appropriate dependence relations between the target and source, and hence the Dependence Thesis is borne out. Finally, the fourth possibility is that the cases are genuine counterexamples, and so Dependence is false.

Below I'll explain what I take to be the problems with the first three responses. Each of these views could, of course, be discussed at great length, and I don't claim to settle the issue once and for all. But I hope to show that it's at best unclear how to maintain Dependence in the face of the above cases.

\subsection{The first possibility: denying explanatoriness}

The first approach to saving the Dependence Thesis is to deny that the viewing-as cases really involve explanation. One can imagine this stance emanating either from skepticism about mathematical explanation in general, or from worries about these cases in particular. I don't intend to say much about the first possibility - although a very few voices of doubt have been raised about the existence of mathematical explanations (notably [Zelcer 2013]), the arguments offered on this score have been unconvincing, and at any rate I think the last fifteen years of research have shown that there's a phenomenon here worth theorizing about. ${ }^{15}$ As for the second possibility, it's not clear what features of the above examples would mark them as especially problematic. There's nothing, after all, to suggest that explanation-talk is being used lightly or figuratively in the relevant passages. And in the absence of such warning signs, I think philosophers should default to taking mathematicians' accounts of their practice seriously, especially when those accounts fall into a coherent pattern indicative of a widely shared standard. So, at least pending further argument, this line of thought doesn't look promising.

A different version of this strategy is to accept mathematicians' judgments about the viewing-as cases, but to insist at the same time that mathematical explanations are essentially different from explanations in the standard sense. One could then interpret the Dependence Thesis as only applying to (ordinary) explanations, so that it can't possibly be falsified by mathematical examples.

The problem with taking this line is that it's quite ad hoc. There's no independent reason to think that mathematical explanations aren't explanations in the usual sense. Cases from mathematics may be distinctive in certain ways, but they're not so bizarre and aberrant that they demand metaphysical quarantine. (On the contrary, recent work in philosophy of science suggests that mathematical and scientific explanations often aren't so different at all; cf. [Lange 2016].) Nor is there any evidence that mathematicians generally mean to use explanation-language in a punning, metaphorical or otherwise nonstandard way. So I don't think the Dependence issue can be solved by denying the relevance of mathematical examples.

\footnotetext{
${ }^{15}$ See [Weber \& Frans 2017] for a reply to Zelcer.
} 


\subsection{The second possibility: relocating the explanantia}

The second argument in defense of the Dependence Thesis rests on the claim that, although the viewing-as cases really do involve explanations of facts about the source objects, the explanantia in these cases are something other than the target objects. In order to preserve Dependence, of course, the true explanantia will have to be things that do bear determination relations to the source objects.

What might these be? One option is that it's the proofs figuring in these cases that ultimately explain, and also metaphysically ground or determine, the relevant facts about the source objects. This view has a certain weight of tradition behind it: starting with Mark Steiner's work in the 1970s, there's been a tendency to think (or at least assume) that all cases of mathematical explanation involve explanatory proofs in some essential way. If that were true, then this strategy would have some degree of initial plausibility. As [D'Alessandro 2018] argues, however, such "proof chauvinist" assumptions are dubious - there seem to be many perfectly good cases of mathematical explanation in which proofs play no important role. So there's no a priori reason to expect they'll solve the dependence problem.

What's more, there's good reason to think that proofs can't play this role. The hypothesis gets the direction of dependence wrong, for one. Surely it's mathematical objects and properties that are metaphysically fundamental, and representations of those objects and properties, like proofs, that are derivative. The idea that proofs "make" or are "responsible for" facts about mathematical objects is about as plausible as the claim that quarks are made or determined by papers in physics journals.

In any case, there's an alternative way to spell out the second argument. Perhaps the explanantia in the viewing-as cases are really facts about the source objects themselves. On this picture, facts about the target objects don't directly contribute anything to the relevant explanations. They may serve some sort of purpose - making an explanation more obvious, or easier to state, or something like that - but they aren't essential. In principle, we could give more or less the same explanation without mentioning the target objects at all. Since there's no mystery about how some properties of an object can be determined by other properties of the very same object, this scenario appears to be consistent with the Dependence Thesis.

The problem with this story is that it's not at all clear how to subtract the target objects from the explanations in the above cases. In what sense could one possibly give "the same" explanation of why the sum of the first $n$ odd numbers is $n^{2}$ without talking about arrays of dots (or whatever)? ${ }^{16}$ This hardly seems possible, since a key component of the explanans is the fact that certain transformations of square arrays of side $n$ yield square arrays of side $n+1$. Similarly, how might we follow Gowers in explaining the appearance of $\pi$ in the Euler identity without mentioning functions defined on the circle, when it's precisely the idea of averaging over such a function that brings $\pi$ into the picture? In these and the other examples, the target objects seem not to function as convenient-but-dispensable accessories. Rather, it looks like the explanations in these cases depend in a constitutive and direct way on facts about the target objects.

\subsection{The third possibility: identifying dependence relations}

Finally, consider the third strategy for defending the Dependence Thesis. This line of thought admits that facts about the target objects explain facts about the source objects, but also insists that the former bear appropriate determinative relations to the latter, as required by Dependence. Since these cases don't feature any familiar forms of causal or metaphysical dependence, of course, the proponent of the third strategy will have to explain which relations she has in mind.

The recent literature contains a couple of proposals along these lines. [Pincock 2015], for instance, argues that some proofs are explanatory because the objects appearing in the proofs stand in a particular metaphysical relation to the objects appearing in the associated theorems. Pincock calls this relation "abstract dependence". (This notion was briefly mentioned in §1.)

Pincock's main motivating example is the relationship between polynomial equations and their Galois groups, as described by Galois theory. It's long been known that a given polynomial equation is "solvable in radicals"-i.e., that there exists a formula expressing the equation's solutions in terms of its coefficients, basic arithmetical operations and $n$th roots - just in case the polynomial's Galois group has a certain algebraic property, also known as "solvability". There's also widespread agreement that this is a case of

\footnotetext{
${ }^{16}$ Obviously there's nothing special about dots in particular-we could just as well use arrays of stars or triangles, say. What's essential to the explanation is the configuration of the arrays, not the identities of the individual elements.
} 
mathematical explanation: the Galois-theoretic criterion explains why certain equations (but not others) are solvable in radicals. Why should this be? Pincock suggests that "what makes a given polynomial equation solvable.... is that its Galois group is solvable" (11). That is, we have an explanatory relation in this case because the explanandum-objects abstractly depend on the explanans-objects.

Pincock doesn't claim that all cases of mathematical explanation, or even any particular class of cases, involve abstract dependence. So whether or not the viewing-as cases are examples of this type isn't diagnostic of the rightness or wrongness of Pincock's view. But it's worth asking whether abstract dependence might be the sort of determination relation that the Dependence theorist is looking for.

So what, then, is abstract dependence? The details of Pincock's account are somewhat involved, but what matters most for our purposes is that an object $X$ abstractly depends on an object $Y$ only if $Y$ is more abstract than $X$. Relative abstractness, in turn, is analyzed in terms of instancehood, in roughly the type-token sense. For example, the word-type cat has particular token inscriptions of the word 'cat' as instances. So the word-type is more abstract than the word-token, on Pincock's view. Similarly, the "concrete" group $(\{0,1\},+)$ is an instance of the "abstract" cyclic group $C_{2}$. So abstract groups (in particular, the Galois groups of polynomials) count as more abstract than concrete groups (in particular, the automorphism groups of extensions of $\mathbb{C}$ associated with these polynomials). Relative abstractness is only a necessary condition for abstract dependence - a further requirement is $Y$ 's being the least more abstract object than $X$. But it's enough for our purposes to ask whether the viewing-as cases satisfy even the relative abstractness condition.

It's clear that the answer is no. None of the cases discussed above involve anything like a type-token relation between the source and target objects - numbers aren't instances of dot arrays, nor are functions $\mathbb{R} \rightarrow \mathbb{C}$ instances of functions defined on the circle, nor is the hyperbolic plane an instance of the hemisphere of a pseudosphere, nor are complex numbers instances of vectors. So Pincock's abstract dependence isn't a candidate for the dependence relation that holds between the objects in these cases.

Another view that links mathematical explanation to metaphysics can be found in [Lange 2015] (see also chapter 9 of [Lange 2016]). Here Lange compares two types of proofs of Desargues' theorem on intersection points: one type which takes place in the setting of classical Euclidean geometry, and which takes the form of "a motley collection of special cases" ([Lange 2015], 438), and another type which achieves a unified treatment using methods from projective geometry. According to Lange and other commentators, only the second sort of proof is explanatory. Lange presents a theory to account for this - a version of the "explanation as coincidence elimination" approach he employs elsewhere - and he also uses the case to motivate some metaphysical conclusions. It's the latter that I want to consider here.

According to Lange, the fact that projective points can be used to explain theorems of ordinary Euclidean geometry entails that there must be some ontological connection between the two. In particular, such an explanatory relationship can obtain only if projective objects "exist in" Euclidean geometry. Lange summarizes this argument as follows:

P1: Certain facts about points at infinity explain certain facts about Euclidean points, lines and planes. [...]

P2: What explains a fact about some entities must be on an ontological par with those entities.

(Roughly: only facts about what exists can explain facts about what exists.)

C: Points at infinity exist in Euclidean geometry. ([Lange 2015], 461)

What does it mean for a mathematical object to "exist in" a particular mathematical domain? Lange doesn't say much about this, as he seems not to have a settled view. Indeed, he leaves open some fairly radical possibilities, including the view that "what it is for points at infinity to exist in Euclidean geometry is for them to play an explanatory role there" (462). I take it that traditional approaches to ontology regard existence as decidedly different from, and more fundamental than, explanation. So this type of view would involve major revisions to standard metaphysics.

For this reason, it may be worth considering a more conservative interpretation of Lange's proposal. (I don't claim that Lange would or should endorse this interpretation. The view to be described strikes me as a straightforward way of reading his remarks, and as an idea that may have some independent interest. But I'm not suggesting that this is the best Lange could do.) The idea is this. Mathematical objects are divided metaphysically into various collections - let's call them "domains" - such that facts about $X$ can explain facts about $Y$ only if $X$ and $Y$ belong to the same domain. (Perhaps an object can belong to more 
than one domain, so we won't assume that this division is a partition.) " $X$ exists in $D$ " and " $X$ is on an ontological par with $Y$ " are then domain-theoretic statements: the first names a domain that $X$ belongs to, and the second says that $X$ and $Y$ belong to some common domain. This proposal may not give defenders of the Dependence Thesis everything they need, but it gives at least a necessary metaphysical condition on facts about $X$ explaining facts about $Y$.

I have three comments about this interpretation of "existing-in". First, the domains it posits probably won't look much like the familiar classifications of mathematical objects (since there's so much "impure" mathematical explanation cutting across traditional subject boundaries ${ }^{17}$, and there probably won't be more than a few distinct domains (for the same reason). So it's unclear how interesting the resulting metaphysical structure will be. Second, even if the proposal is correct, it still gives us little insight into the nature of the dependence relation we were looking for. After all, the claim that $X$ and $Y$ belong to the same domain doesn't tell us anything about whether $X$-facts explain $Y$-facts, or vice versa, or both, or neither. In particular, belonging to the same domain isn't sufficient for instantiating relations of explanation or dependence. (Presumably the integers 33 and -2766018 belong to the same domain, but I doubt that either one determines or explains anything about the other.) Finally, the proposal raises some awkward metaphysical questions. Why should there be domains of this sort in the first place? In virtue of what does a particular mathematical object belong or not belong to a particular domain? It would be mysterious if the existence and composition of domains were brute ontological facts, but it's also unclear what other facts we could appeal to for an explanation. I conclude that this way of developing Lange's suggestion is neither very appealing nor, even if true, likely to be of much use in defending the Dependence Thesis.

So where does the third strategy stand? The idea, again, was to identify a determinative metaphysical relation between the source and target objects in the viewing-as cases discussed above. We saw previously that none of the familiar grounding relations is a good candidate. And I've just now argued that Pincock's abstract dependence and Lange's "coexistence" proposal don't seem obviously helpful either. (Again, this isn't a criticism of either author, since neither set out to deal with this particular issue.) Of course, it's possible that nobody has discovered the true relation yet. But I don't see much reason to hold out hope. There simply doesn't seem to be any interesting metaphysical theme running through the relevant cases. (What do the pairs (numbers, dot arrays), (hyperbolic planes, hemispheres of pseudospheres), and (periodic functions $\mathbb{R} \rightarrow \mathbb{C}$, functions defined on the unit circle) have in common, metaphysically speaking?) I conclude that the Dependence Thesis is, at best, a troubled and unpromising claim about the metaphysics of explanation. Perhaps one of the above responses could be developed more successfully. But until someone shows how, the likeliest bet is that the viewing-as cases are genuine counterexamples.

\section{Fictional models as viewing-as explanations}

An important issue raised by the argument I've just given is whether there are legitimate viewing-as explanations in the sciences and elsewhere. If not, then this difference needs to be accounted for somehow - why should a certain style of explanation be permissible in one realm but not others? On the other hand, if scientists do accept viewing-as explanations, how does this acceptance square with the widespread endorsement of the Dependence Thesis in the philosophy of science?

Perhaps the most natural way to approach this question is through the lens of models. After all, thinking about a scientific entity or phenomenon in terms of a model is in many ways akin to viewing one mathematical entity as another. Both practices involve the use of one object (or system of objects) as lens through which to examine, represent and understand another object of interest. (As Elisabeth Camp claims, "we can illuminate the utility and effects of at least many models by treating them as frames" ([Camp forthcoming], 15); I've suggested that viewing-as involves the same sort of frame-mediated cognition). So questions about viewing-as explanations in science are at least closely related to questions about the explanatoriness of (some kinds of) scientific models.

As for whether models explain, an emerging consensus holds that they can, at least in certain cases. Some kinds of model are claimed to be explanatory because they represent the actual causes or mechanisms responsible for the phenomena in question (cf. [Craver 2006]). This sort of model-explanation

\footnotetext{
${ }^{17}$ As Lange notes himself: "a proof's explanatory power is distinct from its 'purity' in the rough sense of its making use of no concepts foreign to the concepts in the theorem being proved" ([Lange 2016], 292).)
} 
is obviously compatible with the Dependence Thesis. Other models are thought to explain by identifying various kinds of noncausal dependence relations. For instance, [Saatsi \& Pexton 2013] claims that the model of Brown, Enquist and West gives a (plausible) geometric explanation of Kleiber's allometric scaling law: "The model seems to explain by virtue of... [showing] how the scaling exponent counterfactually varies with the dimensionality of organisms. But this explanatory modal information is not easily construed as causal dependence" (620). ${ }^{18}$ Dependence theorists should have no problem with these cases either, since they still involve familiar metaphysical grounding relations.

If Dependence is true, then we should expect all explanatory models to belong to one of these two general types. This is the view espoused by [Povich 2016], who writes that "[models] are explanatory because they accurately represent the relevant dependence relations, that is, the objective features of the world on which the explanandum phenomenon counterfactually depends" (4). ${ }^{19}$ However, explanatory power has sometimes been claimed for other, less Dependence-friendly kinds of models. For instance, Alisa Bokulich has recently argued that some "fictional models" are explanatory, although such models fail to represent the actual world even in an approximate or idealized way. According to Bokulich, a fictional model "explains the explanandum by showing that the counterfactual structure of the model is isomorphic (in the relevant respects) to the counterfactual structure of the phenomenon" ([Bokulich 2011], 43). (This view is further developed in [Bokulich 2012], [Bokulich 2016] and [Bokulich forthcoming].) ${ }^{20}$

Take Bohr's model of the atom. Although the Bohr model with its classical-style electron orbits fundamentally misrepresents reality, Bokulich thinks the model nevertheless explains the spectrum of the hydrogen atom. This is because there's "a pattern of counterfactual dependence of the emission spectrum of hydrogen on the elements represented in Bohr's model" (43). ${ }^{21}$ The similar counterfactual structures of Bohr's model and of actual atoms clearly aren't grounded in any objective worldly dependence. In no sense do the orbits represented by the Bohr model cause, or make, or otherwise metaphysically determine the spectrum of hydrogen (or other features of atoms) - plainly they can't, since nothing like Bohr's orbits exists in reality. The most that can be said is that "Bohr's model is able to correctly answer a number of 'what-if-things-had-been-different questions,' such as how the spectrum would change if the orbits were elliptical rather than circular... This shows that Bohr's model is not simply an ad hoc fitting of the model to the empirical data, as would be the case in a merely phenomenological model" (43).

Is Bokulich right that fictional models can genuinely explain, or must all explanatory scientific models obey Dependence, as Povich suggests? I won't try to settle this question here, but I think either alternative is broadly compatible with my proposal about viewing-as.

On the one hand, if models like Bohr's are explanatory, then the scientific and mathematical realms are alike in allowing viewing-as explanations that aren't backed by dependence relations. ${ }^{22}$ This would make the phenomenon I've described in this paper seem less peculiarly parochial. On the other hand,

\footnotetext{
${ }^{18}$ See also [Berger 1998] for an early account of a geometric-style modeling explanation, and [Rice 2015] for a discussion of noncausal "optimality explanations".

${ }^{19}$ Povich is talking specifically about the "minimal models" of [Batterman \& Rice 2014] here, but the rest of the paper makes it clear that the thesis applies to all models, and indeed to scientific explanations in general.

${ }^{20}$ Bokulich is the only author I'm aware of who explicitly rejects Dependence for reasons similar to the ones I've presented. Whether her "eikonic" conception of explanation is a satisfactory alternative is another question. I have doubts about this, but I can't pursue the issue here.

${ }^{21}$ I find this claim a little puzzling, since it's unclear to me in what sense the spectrum of hydrogen counterfactually depends on the features represented by the Bohr model. Taken at face value, the claim seems false, since the key elements represented by the model don't even exist. Perhaps what Bokulich means is that, if the model had been an accurate representation of atomic structure, then the spectrum would have counterfactually depended on the properties of electron orbits and the like. This is true, but of course we can say the same about any model of anything - if the phenomenon in question had been like the model, then the properties of the phenomenon would have depended on the properties of the elements represented in the model. So this can't be a way to identify explanatory models in particular. Alternatively, Bokulich might mean that if the model were different, then this would have been because the relevant observational data (including the spectrum of hydrogen) had been different-i.e., Bohr wouldn't have proposed the model if it hadn't been empirically adequate. But this is a "backtracking" interpretation of the counterfactual and hence can't be used to determine counterfactual dependence. (Cf. [Lewis 1973].) Perhaps the likeliest story is that Bokulich's official view is given by the earlier statement about "isomorphic counterfactual structures", and the current claim is an accidental misstatement.

${ }^{22}$ Bokulich attributes this explanatoriness to similarity of counterfactual structure, but I'm inclined to think that counterfactual similarity by itself doesn't amount to very much if it fails to yield any epistemic or cognitive benefits. A sketch of an argument: Suppose we want to study a complex phenomenon $P$, and we propose to do so by modeling $P$ with a model $M$. As it turns out, $M$ and $P$ have similar counterfactual structures in certain respects. But $M$ is extremely complex itself-it's so hard to work with that it leads to no new predictions, no better understanding, and so on. Moreover, the elements of $M$ in no way accurately represent the actual components of $P$-it's just a coincidence that the two agree in certain ways.
} 
there's a sense in which Povich is justified in wanting to exclude viewing-as explanations that violate Dependence. After all, one of the distinctive goals of scientific explanation is gaining control over the explanandum phenomenon, or at least knowing what such control would require and to what extent it's possible in principle. (This is the main idea behind mechanism-based theories of scientific explanation.) While viewing-as explanations may improve understanding, reduce surprise, and confer other cognitive benefits, they conspicuously fail to tell us how to manipulate, control or intervene.

This package of features is no problem in the mathematical setting, since control and intervention are nonissues. But it gives scientists good reason to be dubious. Indeed, since viewing-as explanations are unable to perform one of the core functions of scientific explanations - and since there are generally alternative causal or metaphysical explanations that do this job better - it may be reasonable to deny that viewing-as cases even count as members of that category. This is an area where scientists and mathematicians have importantly different interests and expectations. So it's not surprising that the explanatory status of fictional models and the like is controversial, and it's no impediment to rejecting Dependence if viewing-as explanations are accepted in one domain but not the other.

\section{$5 \quad$ Viewing-as, dependence and counterfactuals}

Thus far I've focused on ontic dependence as a possible means of underwriting explanatory facts. But another venerable tradition links explanation to counterfactual dependence. The two approaches aren't equivalent; for instance, as the arguments of [Fine 1995] seem to show, one can have counterfactual dependence in the absence of ontic dependence. ${ }^{23}$ So it's worth asking how the counterfactual theory fares with respect to viewing-as explanations. ${ }^{24}$ (The question is timely, since recent work in philosophy of science suggests that at least some non-causal explanations can be handled in counterfactual terms; see e.g. [Saatsi \& Pexton 2013], [Reutlinger 2016].) A thorough discussion of this question would make for another paper, at least, so I'll limit myself to some preliminary points here.

Following Fine and his successors, I'm inclined to think that ontological dependence, grounding and the associated forms of explanation are hyperintensional, and hence counterfactual dependence isn't generally sufficient for explanation. ${ }^{25}$ So I'll deal here with the necessity question. Is it possible to have genuine explanatory relations in the absence of counterfactual dependence? ${ }^{26}$ In particular, can the viewing-as cases I've discussed be understood in counterfactual terms?

Of course, there's a major problem to be faced before the counterfactual approach can even get off the ground in the mathematical setting. The issue is that the antecedents of the relevant mathematical counterfactuals are necessarily false. So we have to extend the standard Lewis-Stalnaker semantics somehow if we don't want all such counterpossibles to come out trivially true. How should we do that? There are a number of "impossible worlds" proposals on the table, but none that stands out as obviously correct, ${ }^{27}$ a choice has to be made (and defended) before the issue can be settled. (On the other hand, if all counterpossibles really are trivially true - as [Williamson 2007], Chapter 5 argues - then all the mathematical cases of interest will trivially involve counterfactual dependence. But I take it this isn't the sort of vindication that counterfactual theorists are hoping for.)

Alternatively, we could put off choosing an official semantic theory and try to get by on our intuitions. The trouble here is that we don't have sufficiently clear intuitions about most of the cases of interest. Take Numbers-Dots. The relevant counterpossible is this: "If dot arrays formed in the appropriate way didn't make squares, then the sum of the first $n$ odd numbers wouldn't be $n^{2}$." Intuitively, should this be true or

\footnotetext{
Would anyone be tempted to say that $M$ explains $P$ ? Presumably not. To the extent that the Bohr model is plausibly explanatory, this is surely at least in part because it's more vivid, intuitive and easy to work with than other ways of thinking about atomic structure, not just because it makes certain (actually and counterfactually) correct predictions.

${ }^{23}$ The famous example: the existence of Socrates counterfactually depends on the existence of the set $\{$ Socrates\}, but the ontological dependence in this case runs only in the opposite direction.

${ }^{24} \mathrm{I}$ 'm grateful to an anonymous referee for suggesting that I say more about counterfactuals.

${ }^{25}$ For more on the need for hyperintensionality in metaphysics, see [Nolan 2014].

${ }^{26} \mathrm{In}$ asking whether counterfactual dependence is necessary for explanation, I mean to set aside worries about overdetermination and similar phenomena.

${ }^{27}$ For discussion and many references, see [Berto $\backslash\{\} \&$ Jago 2018].
} 
false? It depends on how exactly the change specified by the antecedent is supposed to "ramify" across the rest of mathematics. But our pretheoretic judgments don't give us much guidance on that question.

If anything, it seems likely that the relevant counterpossibles are typically false. Consider the proposal of [Baron et al. 2017], for instance - a recent attempt to make sense of mathematical counterpossibles that's as plausible as anything I'm aware of. One of Baron, Colyvan and Ripley's main examples is the well-known cicada case, due to Alan Baker. They propose to evaluate the counterpossible "If 13 wasn't prime, then North American periodical cicadas wouldn't have 13-year life cycles." Here's a description of their approach:

We should not go too far... we still want to hold fixed as much as we can with respect to the natural numbers. What we're ultimately interested in, recall, are the ramifications of twiddling 13. We are not interested in the ramifications of twiddling any other number. In other words, we want to be able to carry out a "surgical strike" on 13 that enables us to gauge the consequences of altering this number... in as much isolation as possible from alterations to anything else within mathematics. Here's our suggestion: work backwards from the desired twiddle. First, twiddle 13 and hold some portion of the number theory structure fixed. Does a contradiction result? If yes, then relax the amount you've held fixed and re-twiddle. Does a contradiction result? If yes, then relax the amount you've held fixed and re-twiddle. Does a contradiction result? If yes... And so on. Stop when you get to the maximal amount you can hold fixed within mathematics without inducing a contradiction. If there is more than one maximal amount, then pick the maximal amount that interests you, and let the interests be set by your context of evaluation. (7)

Suppose we apply this procedure to NumBers-Dots. What we want to do is "twiddle" the geometric properties of dot arrays while holding fixed as much of the rest of mathematics as we can. In particular, we should presumably hold number theory fixed, since the natural numbers are a completely different collection of objects than the one we're dealing with. So the theorem $\sum_{k=1}^{n}(2 k-1)=n^{2}$ remains intact. Thus, on this approach, it's almost certainly not the case that the sum of the first $n$ odd numbers counterfactually depends on the properties of dot arrays.

Whatever one thinks about Baron-Colyvan-Ripley's specific proposal, it seems certain that any reasonable approach will employ some version of their "surgical strike" strategy. If counterpossibles are going to function as interesting tools for reasoning, the impossibility in their antecedents has to be quarantined in some substantial and principled way. There's room for disagreement about what exactly these principles should look like. But it's very plausible that, whenever $T_{1}$ and $T_{2}$ are distinct mathematical theories dealing with disjoint collections of entities, we should hold $T_{2}$ fixed when entertaining a $T_{1}$-related counterpossible. Doing otherwise is likely to incur unacceptably counterintuitive consequences. (Statements like "If the sets $\mathbb{N}$ and $\mathbb{R}$ were the same size, then triangles would have four sides" should pretty clearly all be false.)

If we follow some procedure along these lines, then NumBERS-Dots and other viewing-as explanations won't turn out to involve counterfactual dependence. I don't know of any alternative approach to counterpossibles that unambiguously gives the opposite result.

\section{Conclusion}

This paper has tried to shed some light on the practice of "viewing one mathematical object as another". As I hope is now clear, there are major challenges and rewards involved in this undertaking, extending across fields including the philosophy of mathematical practice, cognitive theory, metaphysics and philosophy of science. In particular, viewing-as is worth studying for its connection to mathematical explanation and the questions it raises about the nature of explanation in general.

To summarize, the claims I've defended here include the following. First, I've suggested that viewing-as is a standard element of mathematical practice, and I've tried to show that mathematicians often use facts about target objects as explanations of facts about source objects. Second, I've noted that there's a prima facie puzzle about how viewing $X$ as $Y$ could explain anything about $X$, given the apparent lack of familiar dependence relations between mathematical objects of different types. Third, I've 
argued that the conflict between the Dependence Thesis and the viewing-as cases is real, and that it can't be easily resolved in a way that preserves Dependence. (I've also suggested that counterfactual approaches to explanation probably fare no better.) If the arguments I've given are right, then it's time to rethink a major point of orthodoxy in the theory of explanation.

So where do we go from here? What notion of explanation can make sense of viewing-as cases in mathematics - and also, perhaps, fictional models in science - if not one the one captured in the Dependence Thesis? A detailed answer to that question will have to wait. But it seems to me that a return to a "cognitive" or "epistemic" notion of explanation may be called for. On this sort of view, an explanation of a fact $f$ is roughly another fact $f^{*}$ that makes us cognitively better off with respect to $f$ in some suitable sense - by making $f$ more obvious, more intuitive, less surprising, or the like.

Working out the details of such a view will no doubt be challenging. But it's a job we'll have to do if, as I think, Dependence isn't the whole story about explanation. ${ }^{28}$

\section{References}

[Baron et al. 2017]

[Batterman \& Rice 2014]

[Beaney et al. forthcoming]

[Bennett 2017]

[Berger 1998]

[Berto \\{\}\& Jago 2018]

[Bokulich 2011]

[Bokulich 2012]

[Bokulich 2016]

[Bokulich forthcoming]
Baron, Sam, Mark Colyvan and David Ripley. 2017. "How mathematics can make a difference." Philosophers' Imprint 17, 1-19.

Batterman, Robert and Collin C. Rice. 2014. "Minimal model explanations." Philosophy of Science 81, 349-376.

Beaney, Michael, Brenda Harrington and and Dominic Shaw (eds.). Forthcoming. Aspect Perception After Wittgenstein: Seeing-as and Novelty. Routledge: New York.

Bennett, Karen. 2017. Making Things Up. Oxford University Press: New York.

Berger, Ruth. 1998. "Understanding science: Why causes are not enough." Philosophy of Science 65, 306-332.

Berto, Francesco and Mark Jago. 2018. "Impossible worlds." In Edward N. Zalta (ed.), The Stanford Encyclopedia of Philosophy (Fall 2018 edition), forthcoming URL =

$<$ https://plato.stanford.edu/archives/fall2018/entries/impossibleworlds $/>$.

Bokulich, Alisa. 2011. "How scientific models can explain." Synthese $180,33-45$.

Bokulich, Alisa. 2012. "Distinguishing explanatory from nonexplanatory fictions." Philosophy of Science 79, 725-737.

Bokulich, Alisa. 2016. "Fiction as a vehicle for truth: Moving beyond the ontic conception." The Monist 99, 260-279.

Bokulich, Alisa. Forthcoming. "Representing and explaining: The eikonic conception of scientific explanation." Philosophy of Science.

\footnotetext{
${ }^{28}$ This paper started life as a third of my dissertation at the University of Illinois at Chicago; I'm very grateful to my advisor, Daniel Sutherland, and to my other committee members, Mahrad Almotahari, Kenny Easwaran, Dave Hilbert and Marc Lange, for their feedback and support. The comments of an anonymous referee for Philosophical Studies led to some significant additions and (I hope) improvements. I've also benefited from conversations about these issues with Liz Camp, Lisa James, Conor Mayo-Wilson and Lauren Woomer.
} 
[Camp 2008]

[Camp forthcoming]

[Coliva 2012]

[Corfield 2005]

[Craver 2006]

[Craver 2014]

[D'Alessandro 2017]

[D'Alessandro 2018]

[Day \& Krebs 2010]

[Detlefsen 1988]

[Ellenberg 2014]

[Entman 2007]

[Fine 1995]

[Fine 2010]

[Floyd 2010]

[Frans \& Weber 2014]
Camp, Elisabeth. 2008. "Showing, telling and seeing: Metaphor and 'poetic' language." Baltic International Yearbook of Cognition, Logic and Communication 3, 1-24.

Camp, Elisabeth. Forthcoming. "Imaginative frames for scientific inquiry: Metaphors, telling facts and just-so stories." In Peter Godfrey-Smith and Arnon Levy (eds.), The Scientific Imagination. Oxford University Press: New York.

Coliva, Annalisa. 2012. "Human diagrammatic reasoning and seeing-as." Synthese 186, 121-148.

Corfield, David. 2005. "Mathematical kinds, or being kind to mathematics." Philosophica 74, 30-54.

Craver, Carl F. 2006. "When mechanistic models explain." Synthese 153, 355-376.

Craver, Carl F. 2014. "The ontic account of scientific explanation." In Marie I. Kaiser, Oliver R. Scholz, Daniel Plenge, and Andreas Hüttemann (eds.), Explanation in the Special Sciences: The Case of Biology and History. Springer: Dordrecht.

D'Alessandro, William. 2017. "Arithmetic, set theory, reduction and explanation." Synthese, DOI:10.1007/s11229-017-1450-8.

D'Alessandro, William. Forthcoming. "Mathematical explanation beyond explanatory proof." British Journal for the Philosophy of Science, DOI: 10.1093/bjps/axy009.

Day, William and Victor J. Krebs (eds.). 2010. Seeing Wittgenstein Anew: New Essays on Aspect-Seeing. Cambridge University Press: New York.

Detlefsen, Michael. 1988. "Fregean hierarchies and mathematical explanation." International Studies in the Philosophy of Science 3, 97-116.

Ellenberg, Jordan. 2014. How Not to Be Wrong: The Power of Mathematical Thinking. Penguin Books: New York.

Entman, Robert. 2007. "Framing bias: Media in the distribution of power." Journal of Communication 57, 163-173.

Fine, Kit. 1995. "Ontological dependence." Proceedings of the Aristotelian Society 95, 269-290.

Fine, Kit. 2010. "Towards a theory of part." Journal of Philosophy 107, 559-589.

Floyd, Juliet. 2010. "On being surprised: Wittgenstein on aspect-perception, logic, and mathematics." In William Day and Victor J. Krebs (eds.), Seeing Wittgenstein Anew: New Essays on Aspect-Seeing. Cambridge University Press: New York.

Frans, Joachim and Erik Weber. 2014. "Mechanistic explanation and explanatory proofs in mathematics." Philosophia Mathematica 22, 231-248. 
[Giaquinto 2007]

[Gowers 2008]

[Gullberg 1997]

[Hafner \& Mancosu 2005]

[Hanna 1990]

[Harari 2008]

[Herald 2010]

[Kemp \& Mras 2016]

[Kim 1994]

[Konvisser 1986]

[Koslicki 2012]

[Lange 2009]

[Lange 2014]

[Lange 2015]

[Lange 2016]

[Lange 2017]
Giaquinto, Marcus. 2007. Visual Thinking in Mathematics: An Epistemological Study. Oxford University Press: New York.

Gowers, Timothy. 2008. " $\pi$." In Timothy Gowers, June Barrow-Green and Imre Leader (eds.), The Princeton Companion to Mathematics. Princeton University Press: Princeton.

Gullberg, Jan. 1997. Mathematics: From the Birth of Numbers. Norton: New York.

Hafner, Johannes and Paolo Mancosu. 2005. "The varieties of mathematical explanation." In Paolo Mancosu, Klaus Frovin Jørgensen and Stig Andur Pedersen (eds.), Visualization, Explanation and Reasoning Styles in Mathematics, Berlin: Springer, 215-250.

Hanna, Gila. 1990. "Some pedagogical aspects of proof." Interchange 21, 6-13.

Harari, Orna. 2008. "Proclus' account of explanatory demonstrations in mathematics and its context." Archiv für Geschichte der Philosophie 90, 137-164.

Herald, Marybeth. 2010. "Situations, frames, and stereotypes: Cognitive barriers on the road to nondiscrimination." Michigan Journal of Gender and Law 17, 39-55.

Kemp, Gary and Gabriele M. Mras (eds.). 2016. Wollheim, Wittgenstein, and Pictorial Representation: Seeing-as and Seeing-in. Routledge: New York.

Kim, Jaegwon. 1994. "Explanatory knowledge and metaphysical dependence." Philosophical Issues 5, 51-69.

Konvisser, Marc W. 1986. Elementary Linear Algebra with Applications. Ardsley House: New York.

Koslicki, Kathrin. 2012. "Varieties of ontological dependence." In Fabrice Correia and Benjamin Schnieder (eds.), Metaphysical Grounding: Understanding the Structure of Reality. Cambridge University Press: New York.

Lange, Marc. 2009. "Why proofs by mathematical induction are generally not explanatory." Analysis 69, 203-211.

Lange, Marc. 2014. "Aspects of mathematical explanation: Symmetry, unity, and salience." Philosophical Review 123, 485-531.

Lange, Marc. 2015. "Explanation, existence and natural properties in mathematics - A case study: Desargues' theorem." Dialectica 69, $435-472$.

Lange, Marc. 2016. Because Without Cause: Non-Causal Explanations in Science and Mathematics. Oxford University Press: New York.

Lange, Marc. 2017. "Mathematical explanations that are not proofs." Erkenntnis, DOI: 10.1007/s10670-017-9941-z. 
[Laptev \& Rozenfel'd 1996]

[Lewis 1973]

[Linnebo 2008]

[Maddy 2000]

[Mancosu 2008]

[Nolan 2014]

[Pincock 2015]

[Povich 2016]

[Resnik 1981]

[Resnik \& Kushner 1987]

[Reutlinger 2016]

[Reutlinger \& Saatsi forthcoming] Reutlinger, Alexander and Juha Saatsi (eds.). Forthcoming. Explanation Beyond Causation: Philosophical Perspectives on Non-Causal Explanations. Oxford University Press: New York.

[Rice 2015]

[Ruben 1990]

[Saatsi \& Pexton 2013]

Laptev, B.L. and B.A. Rozenfel'd. 1996. "Geometry." In A.N. Kolmogorov and A.P. Yushkevich (eds.), Mathematics of the 19th Century: Geometry, Analytic Function Theory. Translated by Roger Cooke. Birkhäuser Verlag: Basel.

Lewis, David. 1973. "Causation.” Journal of Philosophy 70, 556-567.

Linnebo, Øystein. 2008. "Structuralism and the notion of dependence." Philosophical Quarterly 58, 59-79.

Maddy, Penelope. 2000. "Mathematical progress." In Emily Grosholz and Herbert Breger (eds.), The Growth of Mathematical Knowledge. Kluwer: Dordrecht, 341-352.

Mancosu, Paolo. 2008. "Mathematical explanation: Why it matters." In Paolo Mancosu (ed.), The Philosophy of Mathematical Practice, New York: Oxford University Press, 134-150.

Nolan, Daniel. 2014. "Hyperintensional metaphysics." Philosophical Studies 171, 149-160.

Pincock, Christopher. 2015. "The unsolvability of the quintic: A case study in abstract mathematical explanation." Philosophers' Imprint 15, $1-19$.

Povich, Mark. 2016. "Minimal models and the generalized ontic conception of scientific explanation." British Journal for the Philosophy of Science 0, 1-21.

Resnik, Michael. 1981. "Mathematics as a science of patterns: Ontology and reference." Nô̂s 15, 529-550.

Resnik, Michael D. and David Kushner. 1987. "Explanation, independence and realism in mathematics." British Journal for the Philosophy of Science 38, 141-158.

Reutlinger, Alexander. 2016. "Is there a monist theory of causal and non-causal explanations? The counterfactual theory of scientific explanation." Philosophy of Science 83, 733-745.

Rice, Collin C. 2015. "Moving beyond causes: Optimality models and scientific explanation." Nô̂s 49, 589-615.

Ruben, David-Hillel. 1990. Explaining Explanation. Routledge: New York.

Saatsi, Juha and Mark Pexton. 2013. "Reassessing Woodward's account of explanation: Regularities, counterfactuals, and noncausal explanations." Philosophy of Science 80, 613-624. 
[Salmon 1984]

[Salmon 1989]

[Sandborg 1998]

[Schaffer 2016]

[Seymour 2016]

[Shapiro 1997]

[Steiner 1978]

[Tappenden 2008]

[Tversky \& Kahneman 1981]

[Weber \& Frans 2017]

[Williamson 2007]

[Wittgenstein 2009]

[Zelcer 2013]
Salmon, Wesley. 1984. "Scientific explanation: Three basic conceptions." PSA: Proceedings of the Biennial Meeting of the Philosophy of Science Association 2, 293-305.

Salmon, Wesley. 1989. Four Decades of Scientific Explanation. University of Pittsburgh Press: Pittsburgh.

Sandborg, David. 1998. "Mathematical explanation and the theory of why-questions." British Journal for the Philosophy of Science 49, 603-624.

Schaffer, Jonathan. 2016. "Grounding in the image of causation." Philosophical Studies 173, 49-100.

Seymour, P. 2016. "Hadwiger's conjecture." In J. Nash and M. Rassias (eds.), Open Problems in Mathematics. Springer: Berlin.

Shapiro, Stewart. 1997. Philosophy of Mathematics: Structure and Ontology. Oxford University Press: New York.

Steiner, Mark. 1978. "Mathematical explanation." Philosophical Studies $34,135-151$.

Tappenden, Jamie. 2008. "Mathematical concepts: Fruitfulness and naturalness." In Paolo Mancosu (ed.), The Philosophy of Mathematical Practice. Oxford University Press: New York.

Tversky, Amos and Daniel Kahneman. 1981. "The framing of decisions and the psychology of choice." Science 211, 453-458.

Weber, Erik and Joachim Frans. 2017. "Is mathematics a domain for philosophers of explanation?" Journal for General Philosophy of Science $48,125-142$.

Williamson, Timothy. 2007. The Philosophy of Philosophy. Blackwell: Oxford.

Wittgenstein, Ludwig. 2009. Philosophical Investigations (fourth edition). Translated by G.E.M. Anscombe, P.M.S. Hacker and Joachim Schulte. Wiley-Blackwell: Oxford.

Zelcer, Mark. 2013. "Against mathematical explanation." Journal for General Philosophy of Science 44, 173-192. 